\title{
Energy Source Allocation Decision-Making in Textile Industry: A Novel Symmetric and Asymmetric Spherical Fuzzy Linear Optimization Approach
}

\author{
Sajida Kousar $\mathbb{D}^{1},{ }^{1}$ Urooj Shafqat $\left(\mathbb{D},{ }^{1}\right.$ Nasreen Kausar ${ }^{(D},{ }^{2}$ Dragan Pamucar $\mathbb{D}^{\circ},{ }^{3}$ \\ and Yaé Ulrich Gaba $\mathbb{D I}^{4,5,6}$ \\ ${ }^{1}$ Department of Mathematics and Statistics, International Islamic University Islamabad, Pakistan \\ ${ }^{2}$ Department of Mathematics, Faculty of Arts and Sciences, Yildiz Technical University, Esenler, 34210, Istanbul, Turkey \\ ${ }^{3}$ Department of Logistics, University of Defence in Belgrade, Belgrade, Serbia \\ ${ }^{4}$ Quantum Leap Africa (QLA), AIMS Rwanda Center, Remera Sector KN 3, Kigali, Rwanda \\ ${ }^{5}$ Institut de Mathématiques et de Sciences Physiques (IMSP/UAC), Laboratoire de Topologie Fondamentale, \\ Computationnelle et leurs Applications (Lab-ToFoCApp), BP 613, Porto-Novo, Benin \\ ${ }^{6}$ African Center for Advanced Studies, P.O. Box 4477, Yaounde, Cameroon
}

Correspondence should be addressed to Yaé Ulrich Gaba; yaeulrich.gaba@gmail.com

Received 3 November 2021; Revised 20 December 2021; Accepted 17 January 2022; Published 16 February 2022

Academic Editor: Akif Akgul

Copyright (C) 2022 Sajida Kousar et al. This is an open access article distributed under the Creative Commons Attribution License, which permits unrestricted use, distribution, and reproduction in any medium, provided the original work is properly cited.

In this study, the authors extended the concept of spherical fuzzy optimization models by considering different parameters of spherical fuzzy linear programming problem as symmetric and asymmetric spherical numbers. Eight spherical fuzzy linear programming models are discussed by converting decision variables, parameters, and coefficients of objective function and constraints into symmetric and asymmetric spherical fuzzy numbers. To verify the validity and efficiency of this study in contrast with a linear programming numerical and a physical energy optimization model for the textile industry is considered. The application of these symmetric and asymmetric spherical fuzzy optimization models is discussed along with the postoptimal analysis of the best optimization models that provide the feasible and most optimal solution.

\section{Introduction}

Growing urbanization is directly related to the increase in energy demands, usage, and cost. Energy optimization is globally targeted by every sector. Mostly, an industrial sector is the one who consumed most of the produced or natural energy. The key factor of rising production cost in the industrial sector is abrupt energy usage. Since industrial sectors cannot ignore this factor, getting help through mathematical modeling such as optimizing cost, profits, loss, and energy for such matters is sane act. A lot of work is performed for the optimal utilization of energy in different areas as Wang et al. presented their general guidelines regarding energy optimization in iron and steel industry by using mass-thermal network optimization [1]. Ullah et al. presented bio-inspired energy optimization techniques with the purpose of power scheduling in an office [2]. According to Ozturk et al., energy consumption could be decreased by using the waste-heat recovery systems for the industrial sector so they presented eighty-five techniques for the reduction of energy consumption in their study where thirteen of them were prioritized and applied as energy-efficient techniques [3]. Kimutai [4] proposed the physical energy optimization model for the textile industry and optimized the energy cost by using linear programming (LP). In the manufacturing sector, textile industries are considered major energy consumption units globally due to their several production stages. In the textile industry, mostly electricity and fuel, such as charcoal and petroleum, are used to create all the required kinds of energy. In Pakistan, this particular 
industrial sector is having a great share up to $8.5 \%$ towards GDP (gross domestic product) and considered Asia's 8th largest textile exporter [5]. Pakistan is having a huge textile industrial sector and now facing many uncertainties due to the unpredicted policy shift 2020-25 and COVID. According to National Electric Power Regulatory Authority's (NEPRA) report, one energy unit fluctuation cost causes almost 4 to 5 hours closure in the production of textile's products [6]. To overcome this loss, it is best to optimize the usage and wastage of energy as much as possible. The most extensively adopted procedure for the optimal solution of modeled problem was linear programming (LP) due to its easy applicable nature that was first introduced by Kantorovich [7]. Advancement in this traditional LP generated several extensions such as bi-level LP, multilevel LP, and multiobjective. These LP extensions are highly applicable in real life such as it gave optimized solutions for transportation, supply chain, energy, profit, loss, and cost optimization problems.

Huge modification happened in linear programming after the introduction of fuzzy sets by Zadeh [8]. Fuzzy linear programming was introduced by Zimmerman [9], who originated the technique to solve the multiobjective linear programming in a fuzzy environment. This method was defined according to the natural environmental uncertainties as all the optimization conditions can be considered in fuzzy. Improvements are continuously occurring till now; firstly, membership degree was considered well enough to understand decision-makers choices, but Atanssove [10] created an intuitionistic fuzzy set dealing with the degree of membership and nonmembership clearly, recognizing the choice of an element from the decision set. This definition of the intuitionistic fuzzy set became another reason for improvement in optimization techniques, and firstly, intuitionistic fuzzy (IF) optimization got revealed by Angelov [11]. A lot of work has been carried out in intuitionistic fuzzy linear programming (IFLP). Afterwards, Yager presented the concept of another generalization of fuzzy sets and named it Pythagorean fuzzy set by refining the condition that membership and nonmembership can be independent of each other and their sum of squares must be less than 1 [12]. In 1999, Smarandache [13] introduced a neutrosophic set, which covers the third predictable choice of decision- makers that might be neutral or indeterminacy. In the neutrosophic environment, many optimization models were considered and solved by Ahmad et al. [14, 15].

Recently, a spherical fuzzy set has been introduced by Gundogdu and Kahraman [16]. A spherical fuzzy set is defined with the compliance of positive, neutral, and negative membership functions under the condition that the sum of their squares must be less than 1 providing more general way to cope with uncertainty. It is considered that a spherical fuzzy set is a superset of fuzzy, Pythagorean fuzzy, and intuitionistic fuzzy sets. Ahmad and Adhami [17] presented their work on spherical fuzzy linear programming problem (SFLPP). They presented different types of optimization models under the spherical fuzzy (SF) environment. In this study, we are presenting symmetric and asymmetric energy optimization models inspired by the work of Ahmad and Adhami [17]. For this purpose, the LP model for the textile industry is considered in the spherical fuzzy environment as a numeric example to validate the working of generated energy optimization models in the SF environment. For the conversion of LP into SFLP, parameters were considered spherical fuzzy numbers (SFNs). By targeting each parameter one by one, different SF optimization models are constructed. Every model further contains two submodels in it on the basis of symmetric spherical fuzzy number (SSFN) and asymmetric spherical fuzzy numbers (ASFN) parameters. The deterministic version corresponding to SFNs is based on the spherical fuzzy set theory. Conclusions are based on the application of these spherical fuzzy models on the energy optimization model. The postoptimal analysis of the best feasible optimized SF model is also discussed.

\section{Preliminaries}

A spherical fuzzy set (SFS) is defined by Rafiq et al. [18] as the following set:

$$
\left\{\left\langle u, p_{\widetilde{S F}}(u), n_{\widetilde{S F}}(u), f_{\widetilde{S F}}(u)\right\rangle: u \in U\right\} .
$$

Considering $U$ the universal discourse and $\widetilde{S F}$ representing spherical fuzzy set such that

$$
\begin{aligned}
& p_{\widetilde{S F}}: U \longrightarrow[0,1], \\
& n_{\widetilde{S F}}: U \longrightarrow[0,1], \\
& f_{\widetilde{S F}}: U \longrightarrow[0,1] \text { with } 0 \leq p_{\widetilde{S F}}^{2}(u)+n_{\widetilde{S F}}^{2}(u)+f_{\widetilde{S F}}^{2}(u) \leq 1 \forall u \in U,
\end{aligned}
$$

where $p_{S F}^{\sim}(u)$ is positive membership degree, and $n_{\widetilde{S F}}(u)$ and $f \sim \mathcal{S F}(u)$ is representing neutral and negative membership degree of each $u \in U$, respectively, to $\widetilde{S F}$. A spherical fuzzy number is a fuzzy number $\widetilde{r}=\left\langle p_{\tilde{r}}, n_{r}, f_{\widetilde{r}}\right\rangle$ with positive, neutral, and negative membership functions defined as 


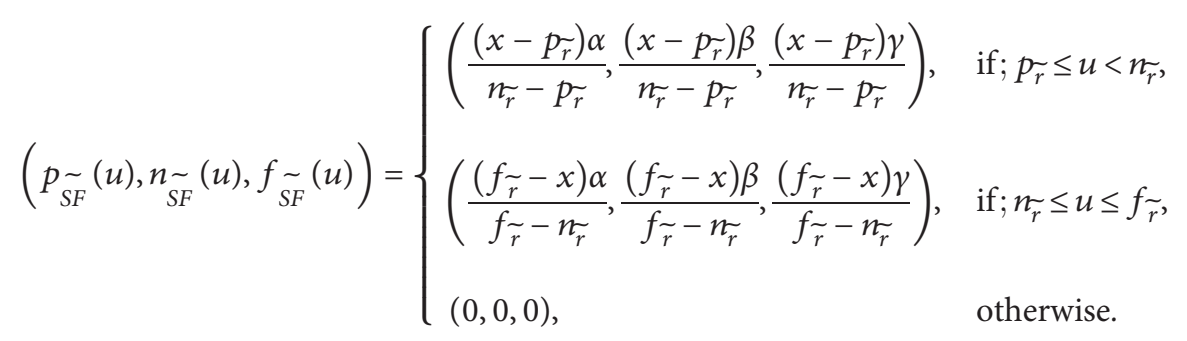

Here, $\alpha, \beta, \gamma \in[0,1]$ such that $0 \leq \alpha^{2}+\beta^{2}+\gamma^{2} \leq 1$. Let $\widetilde{r}=\left\langle p_{\widetilde{r}}, n_{\widetilde{r}}, f_{\widetilde{r}}\right\rangle$ and $\tilde{m}=\left\langle p_{r}, n_{r}, f_{\widetilde{r}}\right\rangle$ are two spherical fuzzy numbers, and then, the algebraic operations [18] between them are defined as follows:

(1) $\tilde{r} \oplus \widetilde{m}=\left\langle\sqrt{p_{\widetilde{r}}^{2}+p_{\widetilde{m}}^{2}-p_{\tilde{r}}^{2} \cdot p_{\widetilde{m}}^{2}}, n_{\widetilde{r}} \cdot n_{\tilde{m}}, f_{\widetilde{r}} \cdot f_{\widetilde{m}}\right\rangle$

(2) $\tilde{r} \circledast \widetilde{m}=\left\langle p_{\tilde{r}}^{\sim} \cdot p_{\tilde{m}}, n_{\tilde{r}} \cdot n_{\widetilde{m}}, \sqrt{f_{\widetilde{r}}^{2}+f_{\widetilde{m}}^{2}-f_{\widetilde{r}}^{2} \cdot f_{\widetilde{m}}^{2}}\right\rangle$

(3) $\kappa \tilde{r}=\left\langle\sqrt{1-\left(1-p_{\tilde{r}}^{2}\right)^{\kappa}},\left(n_{\tilde{r}}\right)^{\kappa},\left(f_{\widetilde{r}}\right)^{\kappa}\right\rangle ; \quad$ for $\kappa \geq 0$

A SFN will be considered symmetric spherical fuzzy number (SSFN) if there exists a relation between positive and neutral, positive, and negative membership. For example,

$$
\begin{aligned}
\widetilde{r} & =\left\langle p_{\widetilde{r}}, n_{\widetilde{r}}, f_{\widetilde{r}}\right\rangle \\
& =\left\langle p_{\widetilde{r}}, p_{\widetilde{r}}+\lambda, p_{\widetilde{r}}+k \lambda\right\rangle,
\end{aligned}
$$

where $\lambda>0, k>1$, and $\lambda, k \in \mathbb{R}$; otherwise, it is considered asymmetric spherical fuzzy number (ASFN).

\section{Spherical Fuzzy Linear Programming Problem}

Ideally for optimal solution of mathematically modeled problem, linear programming (LP) is considered the most convenient way [7]. Since this LP does not accommodate the fuzziness of nature, the best real-life modeled problem solution requires a method of fuzzy optimization. A lot of work is already carried out for fuzzy optimization modeling by utilizing different techniques such as intuitionistic fuzzy linear programming [11, 12] and neutrosophic LP [14-19]. Ahmad and Adhami presented different models for the solution of spherical fuzzy LP [17]. By continuing their idea for SF modeling, different spherical fuzzy models are constructed in this study. In the first model, only constraint coefficients were considered spherical fuzzy numbers, whereas all the other decision variables and parameters are real quantities. In the second model, two factors demand and constraint coefficients are taken as SFNs, while cost is taken as a real number. In the third model, other than decision variables, all the other factors are considered in the spherical fuzzy number, whereas in the fourth model, the cost and demand are in SF numbers. Table 1 is designed to illustrate all these cases.

In Table $1, \widetilde{S^{s s f}}, \widetilde{D^{s s f}}$, and $\widetilde{C^{s s f}}$ are symmetric spherical fuzzy, $\widetilde{S^{a s f}}, \widetilde{D^{a s f}}$, and $\widetilde{C^{a s f}}$ asymmetric spherical fuzzy parameters, and $s, d, c$ real-valued parameters.

\section{Numerical Example}

Consider the following linear programming problem:

$$
\begin{aligned}
& \operatorname{Max} z=5 x+3 y \\
& \text { subjected to, } \\
& 3 x+5 y \leq 15, \\
& 5 x+2 y \leq 10 .
\end{aligned}
$$

The SSF and ASF for the above LP are presented in Table 2.

From Table 3, it is clear that SSF model-I results in the highest optimal solution value. Since the solution of LP is 12.3684 and we are looking for more better feasible solution, all those models whose values are greater; that is, higher than LP output is considered better. Here, in Table 3, we obtain that

$$
\begin{aligned}
\text { LP } & <\text { ASF Model }-I I<\text { SSF Model }-I I<\text { FLP }<\text { IFLP } \\
& <\text { ASF Model }-I<\text { SSF Model }-I,
\end{aligned}
$$

where the remaining models result in a value less than LP solution so these are not considered better than LP. All the models are providing feasible solution, and the best one is provided by SSF model-I as it results in the highest objective output.

\section{Application}

To elaborate the working efficiency of the above-defined SSF and ASF optimization models of our study, we construct an energy optimization model for the textile industry with five stages shown in Figure 1.

Suppose $X_{i}$ is the number of units of product that processed at stage $i$. In the objective function, the cost coefficients are according to the type of energy used for the preparation of per unit product of stage $i . D_{i}$ is the monthly demand of each product $X_{i}$ and availability of working hours that helped to form the following demand constraint equations according to the stages presented in Figure 1:

$$
\begin{aligned}
x_{1}-x_{2} & \geq 400, \\
0.03 x_{2}-x_{3}+0.07 x_{4} & =0, \\
0.97 x_{2}-x_{4} & =0, \\
0.93 x_{4}-x_{5} & \geq 600, \\
0 . .96 x_{5} & \geq 20000, \\
0.007 x_{1}-0.07 x_{2}+0.013 x_{4}+0.0062 x_{5} & \leq 720 .
\end{aligned}
$$

Each stage in Figure 1 is also presenting the production cost per unit of each stage's product along with information about how much quantity is going to be processed further in 
TABLE 1: Spherical fuzzy linear programming models.

\begin{tabular}{|c|c|c|}
\hline Model & Symmetric spherical fuzzy model (a) & Asymmetric spherical fuzzy model (b) \\
\hline I & $\begin{array}{l}\text { Optimize } \alpha=\sum_{i=1}^{K} c_{i} x_{i} \text { subjected to } \\
\sum_{i=1}^{K} \widetilde{S_{i j}^{s s f}} x_{i} \leq=\geq d_{j}, \forall j=1,2,3 \ldots l\end{array}$ & $\begin{array}{l}\text { Optimize } \alpha=\sum_{i=1}^{K} c_{i} x_{i} \text { subjected to } \\
\sum_{i=1}^{K} \widetilde{S_{i j}^{a s f}} x_{i} \leq=\geq d_{j}, \forall j=1,2,3 \ldots l\end{array}$ \\
\hline II & $\begin{array}{c}\text { Optimize } \alpha=\sum_{i=1}^{K} c_{i} x_{i} \text { subjected to } \sum_{i=1}^{K} \widetilde{S_{i j}^{s f} x_{i}} \leq=\geq \widetilde{D_{j}^{s s f}} \\
\forall j=1,2,3 \ldots l\end{array}$ & $\begin{array}{c}\text { Optimize } \alpha=\sum_{i=1}^{K} c_{i} x_{i} \text { subjected to } \\
\sum_{i=1}^{K} \widetilde{S_{i j}^{a s f}} x_{i} \leq=\geq \widetilde{D_{j}^{a s f}}, \forall j=1,2,3 \ldots l\end{array}$ \\
\hline III & $\begin{array}{l}\text { Optimize } \alpha=\sum_{i=1}^{K} \widetilde{C_{i}^{s s f}} x_{i} \text { subjected to } \\
\sum_{i=1}^{K} \widetilde{S_{i j}^{s s f}} x_{i} \leq=\geq \widetilde{D_{j}^{s s f}}, \forall j=1,2,3 \ldots l\end{array}$ & $\begin{array}{l}\text { Optimize } \alpha=\sum_{i=1}^{K} \widetilde{C_{i}^{a s f}} x_{i} \text { subjected to } \\
\sum_{i=1}^{K} \widetilde{S_{i j}^{a s f}} x_{i} \leq=\geq D_{j}^{a s f}, \forall j=1,2,3 \ldots l\end{array}$ \\
\hline IV & $\begin{array}{c}\text { Optimize } \alpha=\sum_{i=1}^{K} \widetilde{C_{i}^{s s f}} x_{i} \text { subjected to } \sum_{i=1}^{K} s_{i j} x_{i} \leq=\geq \widetilde{D_{j}^{s s f}}, \\
\forall j=1,2,3 \ldots l\end{array}$ & $\begin{array}{c}\text { Optimize } \alpha=\sum_{i=1}^{K} \widetilde{C_{i}^{a s f}} x_{i} \text { subjected to } \sum_{i=1}^{K} s_{i j} x_{i} \leq=\geq \widetilde{D_{j}^{a s f}} \\
\forall j=1,2,3 \ldots l\end{array}$ \\
\hline
\end{tabular}

TABLE 2: Spherical fuzzy linear programming models.

\begin{tabular}{|c|c|c|}
\hline Model & Symmetric spherical fuzzy model (a) & Asymmetric spherical fuzzy model (b) \\
\hline I & $\operatorname{Max} Z=5 x+3 y$ subjected to $\widetilde{3^{s s f}} x+\widetilde{5^{s s f}} y \leq 15$ & $\operatorname{Max} Z=5 x+3 y$ subjected to $\widetilde{3^{\text {asf }} x} x+\widetilde{5^{\text {asf }}} y \leq 15$ \\
\hline II & $\operatorname{Max} Z=5 x+3 y$ subjected to $\widetilde{3^{s s f}} x+\widetilde{5^{s s f}} y \leq \widetilde{15^{s s f}}$ & $\operatorname{Max} Z=5 x+3 y$ subjected to $\widetilde{3^{a s f}} x+\widetilde{5^{a s f}} y \leq \widetilde{15^{a s f}}$ \\
\hline III & $\operatorname{Max} Z=\widetilde{5^{s s f}} x+\widetilde{3^{s s f}} y$ subjected to $\widetilde{3^{s s f}} x+\widetilde{5^{s s f}} y \leq \widetilde{15^{s s f}}$ & $\operatorname{Max} Z=\widetilde{5^{a s f}} x+\widetilde{3^{a s f}} y$ subjected to $\widetilde{3^{a s f}} x+\widetilde{5^{a s f}} y \leq \widetilde{15^{\text {asf }}}$ \\
\hline IV & $\operatorname{Max} Z=\widetilde{5^{s s f}} x+\widetilde{3^{s s f}} y$ subjected to $3 x+5 y \leq \widetilde{15^{s s f}}$ & $\operatorname{Max} Z=\widetilde{5^{\text {asf }}} x+\widetilde{3^{\text {asf }}} y$ subjected to $3 x+5 y \leq \widetilde{15^{\text {asf }}}$ \\
\hline
\end{tabular}

TABLE 3: Comparison of LP and different fuzzy optimization models for the numerical example.

\begin{tabular}{lccc}
\hline Optimization model & Objective function & $x$ & $y$ \\
\hline LP & 12.3684 & 1.05263 & 2.36842 \\
FLP & 15.13159 & 1.44737 & 2.63158 \\
IFLP & 16.31579 & 1.052632 & 2.368421 \\
SSF Model-I & 22.1171 & 3.119093 & 2.173913 \\
ASF Model-I & 16.58654 & 1.442308 & 3.125 \\
SSF Model-II & 12.97593 & 1.100301 & 2.491472 \\
ASF Model-II & 12.71875 & 1.0625 & 2.46875 \\
SSF Model-III & 9.691474 & 1.100301 & 2.491472 \\
ASF Model-III & 8.957813 & 1.0625 & 2.46875 \\
SSF Model-IV & 6.808947 & 0.684211 & 1.8894744 \\
ASF Model-IV & 6.660789 & 0.768421 & 1.878947 \\
\hline
\end{tabular}

the next stage by considering electricity cost $20.62 \mathrm{PKR} /$ $\mathrm{kWh}$, fuel (furnace oil) price PKR85.68/litre, and LPG cost at the rate of PKR19.4103/litre [20,21]. The last constraint is regarding availability of total working time and production rate, that is, how much hours are needed for the production of $1 \mathrm{~kg}$ product of spinning, weaving, and final stages. Objective function constructed through Figure 1 is

$$
\begin{aligned}
\min z= & 51.55 x_{1}+24.25653 x_{2}+41.24 x_{3}+15.465 x_{4} \\
& +177.8921 x_{5} .
\end{aligned}
$$

To conduct optimization in the spherical fuzzy environment, the uncertainty in demand, supply parameters, and energy cost per unit fluctuation is kept in mind and considered symmetric and asymmetric SFNs. The considered positive, neutral, and negative membership degree of acceptance is throughout $(0.5,0.3,0.2)$.

\section{Model-I(a)}

Optimize $\alpha=\sum_{i=1}^{5} c_{i} x_{i}$ subjected to $\sum_{i=1}^{5} \widetilde{S_{i j}^{s f f}} x_{i} \leq \equiv \geq d_{j}$, $\forall j=1,2, \ldots, 5$, where spherical fuzzy coefficients $S_{i j}^{s s f}$ are considered symmetric with $x_{i}$ real decision variables where $d_{j}$ is real-valued demands. After the conversion of an inconsideration energy optimization model for the textile industry into the symmetric spherical fuzzy model-I(a), it is represented as follows:

Cost set is $C=\left\{c_{i}, i=1,2,3,4,5\right\} C=$ $\{51.55,24.5653,41.24,15.46,177.8921\}$, where symmetric spherical fuzzy constraint coefficients are 


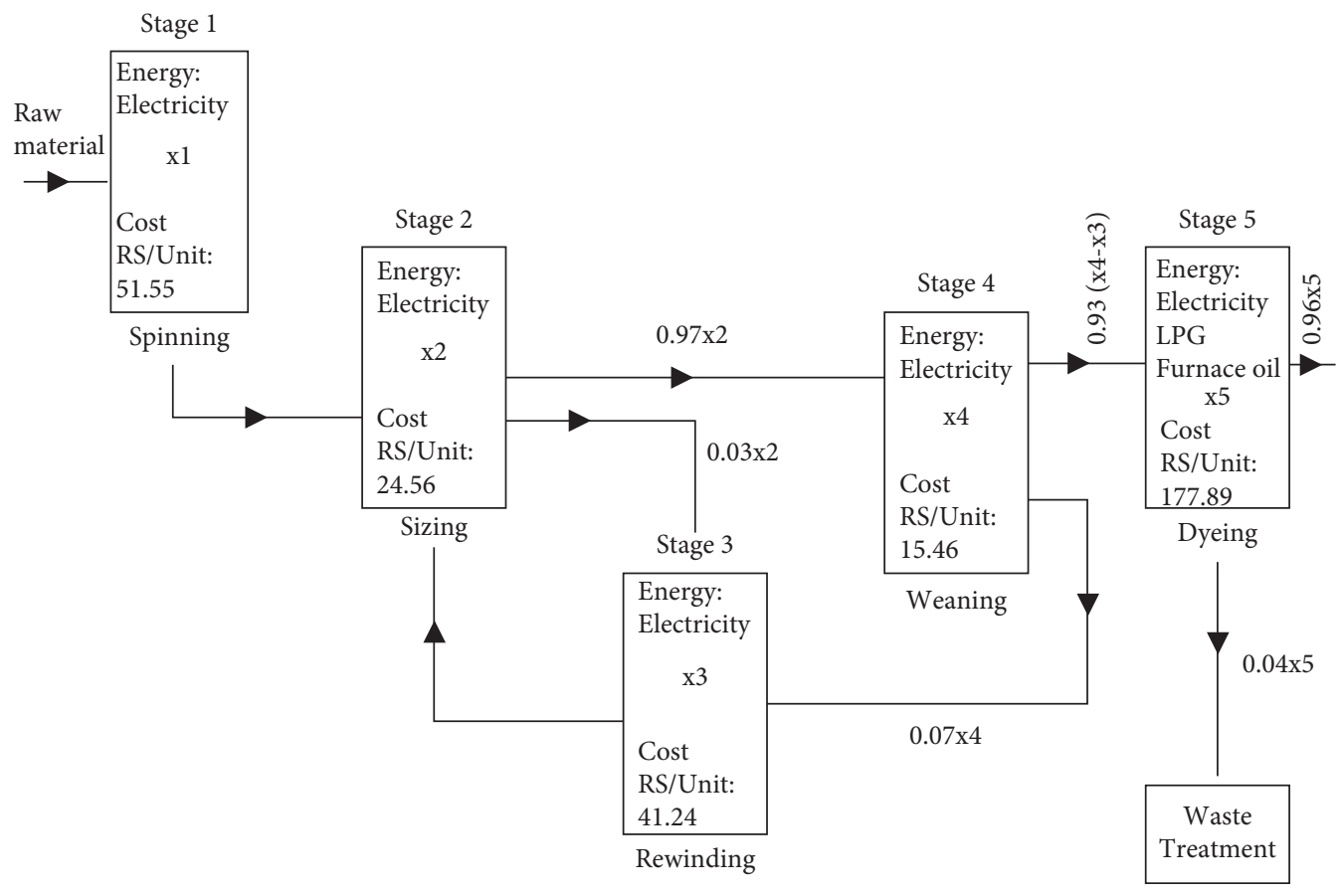

Figure 1: Production stages.

$\widetilde{S_{21}^{s f f}}=(0.03)^{s s f}=(0.03,0.08,0.13)$,

$\widetilde{S_{23}^{s f f}}=(0.007)^{s s f}=(0.007,0.012,0.017)$,

$\widetilde{S_{31}^{s s f}}=(0.97)^{s s f}=(0.97,1.02,1.07)$,

$\widetilde{S_{41}^{s s f}}=(0.93)^{s s f}=(0.93,0.98,1.03)$,

$\widetilde{S_{51}^{s s f}}=(0.96)^{s s f}=(0.96,1.01,1.06)$,

$\widetilde{S_{63}^{s s f}}=(0.013)^{s s f}=(0.013,0.018,0.023)$,

$\widetilde{S_{64}^{s s f}}=(0.0062)^{s s f}=(0.0062,0.0067,0.0072)$,

$\widetilde{S_{i j}^{s s f}}=(1)^{s s f}$

$=(1,0,0) \cdot$ for all the values of $\$ i j \$$ other than above. with the monthly production demand in $\mathrm{kg}$ for three products and total availability of working hours in a month as $D=\left\{d_{1}=400, d_{2}=600, d_{3}=20000, d_{4}=720\right\}$. Mathematically, symmetric spherical fuzzy energy optimization is framed as follows:

$$
\begin{aligned}
\min \widetilde{\alpha^{v}}= & \sum_{j=1}^{m} c_{j} x_{j} \\
= & 51.55 x_{1}+24.25653 x_{2}+41.24 x_{3}+15.465 x_{4} \\
& +177.8921 x_{5} .
\end{aligned}
$$

Subjected to

$$
\begin{aligned}
x_{1}-x_{2} & \geq 400, \\
(0.03)^{s s f} x_{2}-x_{3}+(0.07)^{s s f} x_{4} & =0, \\
(0.97)^{s s f} x_{2}-x_{4} & =0, \\
(0.93)^{s s f} x_{4}-x_{5} & \geq 600, \\
(0.96)^{s s f} x_{5} & \geq 20000, \\
(0.007)^{s s f} x_{1}-(0.007)^{s s f} x_{2}+(0.013)^{s s f} x_{4}+(0.0062)^{s s f} x_{5} & \leq 720, \\
x_{i} & \geq 0, \quad i=1,2,3,4,5 .
\end{aligned}
$$


The above symmetric spherical fuzzy liner programming model is then converted into LP, and the following defuzzified constraints are obtained:

$$
\begin{aligned}
x_{1}-x_{2} & \geq 400, \\
0.065 x_{2}-x_{3}+0.0105 x_{4} & =0, \\
1.005 x_{2}-x_{4} & =0, \\
0.965 x_{4} & \geq 600, \\
0.995 x_{5} & \geq 20000, \\
0.0105 x_{1}-0.0105 x_{2}+0.0165 x_{4}+0.00655 x_{5} & \leq 720, \\
x_{i} & \geq 0, \quad i=1,2,3,4,5 .
\end{aligned}
$$

\section{Model-I(b)}

Now in this model, the spherical fuzzy coefficients $\widetilde{S_{i j}^{a s f}}$ are considered asymmetric with $x_{i}$ real-valued decision variables with $d_{j}, c_{i}$, which are real demands and costs.

For this purpose, by changing the values of spherical fuzzy coefficients by asymmetric spherical fuzzy in the above model-I(a) in (9) for the textile industry, the following changes occurred:

$\widetilde{S_{21}^{\text {asf }}}=(0.03)^{\text {asf }}=(0.03,0.08,1.08)$,

$\widetilde{S_{23}^{\text {asf }}}=(0.007)^{\text {asf }}=(0.007,0.012,1.012)$,

$\widetilde{S_{31}^{a s f}}=(0.97)^{\text {asf }}=(0.97,1.02,2.02)$,

$\widetilde{S_{41}^{a s f}}=(0.93)^{a s f}=(0.93,0.98,1.98)$,

$\widetilde{S_{41}^{a s f}}=(0.96)^{a s f}=(0.96,1.01,2.06)$,

$\widetilde{S_{53}^{a s f}}=(0.013)^{\text {asf }}=(0.013,0.018,1.023)$,

$\widetilde{S_{54}^{a s f}}=(0.0062)^{a s f}=(0.0062,0.0067,1.0072)$

$\widetilde{S_{i j}^{a s f}}=(1)^{a s f}=(1,0,0) \cdot$ for all the values of $\$ i j \$$ other than above.
The monthly production demand in $\mathrm{kg}$ for three products and total availability of working hours in a month are $D=\left\{d_{1}=400, d_{2}=600, d_{3}=20000, d_{4}=720\right\}$. The asymmetric spherical fuzzy energy optimization model is

$$
\begin{aligned}
\min \widetilde{\alpha^{v}}= & \sum_{j=1}^{m} c_{j} x_{j} \\
= & 51.55 x_{1}+24.25653 x_{2}+41.24 x_{3} \\
& +15.465 x_{4}+177.8921 x_{5},
\end{aligned}
$$

subjected to constraints

$$
\begin{aligned}
x_{1}-x_{2} & \geq 400, \\
(0.03)^{a s f} x_{2}-x_{3}+(0.07)^{a s f} x_{4} & =0, \\
(0.97)^{a s f} x_{2}-x_{4} & =0, \\
(0.93)^{a s f} x_{4}-x_{5} & \geq 600, \\
(0.96)^{a s f} x_{5} & \geq 20000, \\
(0.007)^{a s f} x_{1}-(0.007)^{a s f} x_{2}+(0.013)^{a s f} x_{4}+(0.0062)^{a s f} x_{5} \leq 720, & \\
x_{i} & \geq 0, \quad i=1,2,3,4,5 .
\end{aligned}
$$


The defuzzyfied form of the above ASF linear programming model is given as

$$
\begin{aligned}
x_{1}-x_{2} & \geq 400, \\
0.255 x_{2}-x_{3}+0.2095 x_{4} & =0, \\
1.195 x_{2}-x_{4} & =0, \\
1.155 x_{4} & \geq 600, \\
1.185 x_{5} & \geq 20000,
\end{aligned}
$$

$0.2095 x_{1}-0.2095 x_{2}+0.2155 x_{4}+0.20645 x_{5} \leq 720$,

$$
x_{i} \geq 0, \quad i=1,2,3,4,5 \text {. }
$$

\section{Model-II(a)}

In this model, constraint coefficients and demand is considered in the symmetric spherical fuzzy number and cost remains unchanged as follows:

$$
\begin{aligned}
\widetilde{D_{1}^{s s f}} & =(400)^{s s f}=(400,450,500), \\
\widetilde{D_{2}^{s s f}} & =(0)^{s s f}=(0,0,0)=\widetilde{D_{3}^{s s f}}, \\
\widetilde{D_{4}^{s s f}} & =(600)^{s s f}=(600,650,700), \\
\widetilde{D_{5}^{s s f}} & =(20000)^{s s f}=(20000,20050,20100), \\
\widetilde{D_{6}^{s s f}} & =(720)^{s s f}=(720,770,820), \\
\widetilde{S_{21}^{s s f}} & =(0.03)^{s s f}=(400,450,500), \\
\widetilde{S_{23}^{s s f}} & =(0.007)^{s s f}=(400,450,500), \\
\widetilde{S_{31}^{s s f}} & =(0.97)^{s s f}=(400,450,500), \\
\widetilde{S_{41}^{s s f}} & =(0.96)^{s s f}=(400,450,500), \\
\widetilde{S_{53}^{s s f}} & =(0.013)^{s s f}=(400,450,500), \\
\widetilde{S_{54}^{s s f}} & =(0.0062)^{s s f}=(400,450,500), \\
\widetilde{S_{i j}^{s s f}} & =(1)^{s s f}=(1,0,0) \cdot \text { for all the values of } \$ i j \$ \text { other than above. }
\end{aligned}
$$

The symmetric SF energy optimization model-II(a) for

Under the constraints the textile industry becomes

$$
\begin{aligned}
\min \tilde{\alpha^{\gamma}=} & 51.55 x_{1}+24.25653 x_{2}+41.24 x_{3} \\
& +15.465 x_{4}+177.8921 x_{5} .
\end{aligned}
$$

$$
\begin{aligned}
x_{1}-x_{2} & \geq(400)^{s s f}, \\
(0.03)^{s s f} x_{2}-x_{3}+(0.07)^{s s f} x_{4} & =(0)^{s s f}, \\
(0.97)^{s s f} x_{2}-x_{4} & =(0)^{s s f}, \\
(0.93)^{s s f} x_{4}-x_{5} & \geq(600)^{s s f} \\
(0.96)^{s s f} x_{5} & \geq(20000)^{s s f} \\
(0.007)^{s s f} x_{1}-(0.007)^{s s f} x_{2}+(0.013)^{s s f} x_{4}+(0.0062)^{s s f} x_{5} & \leq(720)^{s s f} \\
x_{i} & \geq 0, \quad i=1,2,3,4,5,
\end{aligned}
$$


converting the above constraint into LP:

$$
\begin{aligned}
x_{1}-x_{2} & \geq 435, \\
0.065 x_{2}-x_{3}+0.0105 x_{4} & =0, \\
1.005 x_{2}-x_{4} & =0, \\
0.965 x_{4} & \geq 635, \\
0.995 x_{5} & \geq 20035, \\
x_{i} & \geq 0, \quad i=1,2,3,4,5 .
\end{aligned}
$$

$0.0105 x_{1}-0.0105 x_{2}+0.0165 x_{4}+0.00655 x_{5} \leq 755$,
Solve the objective function 0.13 subjected to set of constraints 0.15 to obtain the solution.

\section{Model-II(b)}

In this model, constraint coefficients and demand is considered asymmetric spherical fuzzy number:

$$
\begin{aligned}
& \widetilde{D_{1}^{a s f}}=(400)^{a s f}=(400,450,511), \\
& \widetilde{D_{2}^{a s f}}=(0)^{a s f}=(0,0,0)=\widetilde{D_{3}^{a s f}}, \\
& \widetilde{D_{4}^{a s f}}=(600)^{a s f}=(600,650,711), \\
& \widetilde{D_{5}^{a s f}}=(20000)^{a s f}=(20000,20050,20111), \\
& \widetilde{D_{6}^{a s f}}=(720)^{a s f}=(720,770,831), \\
& \widetilde{S_{21}^{a s f}}=(0.03)^{a s f}=(400,450,500), \\
& \widetilde{S_{23}^{s s f}}=(0.007)^{s s f}=(400,450,500), \\
& \widetilde{S_{31}^{s s f}}=(0.97)^{s s f}=(400,450,500), \\
& \widetilde{S_{41}^{s s f}}=(0.96)^{s s f}=(400,450,500), \\
& \widetilde{S_{53}^{s s f}}=(0.013)^{s s f}=(400,450,500), \\
& \widetilde{S_{54}^{s s f}}=(0.0062)^{s s f}=(400,450,500), \\
& \widetilde{S_{i j}^{s s f}}=(1)^{s s f}=(1,0,0) \text { for all the values of ij other than above. }
\end{aligned}
$$

The asymmetric SF energy optimization model-II(b) with objective function 0.13 for the textile industry under the constraints:

$$
\begin{aligned}
x_{1}-x_{2} & \geq(400)^{a s f}, \\
(0.03)^{a s f} x_{2}-x_{3}+(0.07)^{a s f} x_{4} & =(0)^{a s f}, \\
(0.97)^{a s f} x_{2}-x_{4} & =(0)^{a s f}, \\
(0.93)^{a s f} x_{4}-x_{5} & \geq(600)^{a s f}, \\
(0.96)^{a s f} x_{5} & \geq(20000)^{a s f}, \\
(0.007)^{a s f} x_{1}-(0.007)^{a s f} x_{2}+(0.013)^{a s f} x_{4}+(0.0062)^{a s f} x_{5} & \leq(720)^{a s f}, \\
x_{i} & \geq 0, i=1,2,3,4,5,
\end{aligned}
$$


converting the above constraint into real-valued:

$$
\begin{aligned}
x_{1}-x_{2} & \geq 437.2, \\
0.255 x_{2}-x_{3}+0.2095 x_{4} & =0, \\
1.195 x_{2}-x_{4} & =0, \\
1.155 x_{4} & \geq 637.2, \\
1.185 x_{5} & \geq 20037.2,
\end{aligned}
$$

$0.2095 x_{1}-0.2095 x_{2}+0.2155 x_{4}+0.20645 x_{5} \leq 727.2$

$$
x_{i} \geq 0, i=1,2,3,4,5 \text {. }
$$

\section{Model-III(a)}

In this model, all the cost and demand coefficients are in SSFNs, so here the objective function is expressed as follows:

$$
\begin{aligned}
\text { Optimize } \alpha= & \sum_{i=1}^{K} \widetilde{C_{i}^{s s f}} x_{i}=\min \widetilde{\alpha^{\vee}}=\sum_{i=1}^{5} \widetilde{C_{i}^{a s f}} \circledast x_{j}, \\
= & \left((51.55,56.55,61.55) \circledast x_{1} \oplus(24.5653,29.5653,34.5653) \circledast x_{2},\right. \\
& \oplus(41.24 \\
& \oplus\left((177.892,182.892,187.892) \circledast x_{5},\right.
\end{aligned}
$$

under the constraints

$$
\begin{aligned}
x_{1}-x_{2} & \geq(400)^{s s f}, \\
(0.03)^{s s f} x_{2}-x_{3}+(0.07)^{s s f} x_{4} & =0 \\
(0.97)^{s s f} x_{2}-x_{4} & =(0)^{s s f}, \\
(0.93)^{s s f} x_{4}-x_{5} & \geq(600)^{s s f}, \\
(0.96)^{s s f} x_{5} & \geq(20000)^{s s f}, \\
(0.007)^{s s f} x_{1}-(0.007)^{s s f} x_{2}+(0.013)^{s s f} x_{4}+(0.0062)^{s s f} x_{5} \leq(720)^{s s f} & x_{i} \geq 0, i=1,2,3,4,5 .
\end{aligned}
$$

after converting the above model into general LP, 0.15 becomes required constraints for the objective function:

$$
\begin{aligned}
\min \tilde{\alpha^{\gamma}=} & 55.05 x_{1}+28.0653 x_{2}+44.74 x_{3}+18.965 x_{4} \\
& +181.3921 x_{5} .
\end{aligned}
$$

\section{Model-III(b)}

By solving objective function,

$$
\begin{aligned}
\text { Optimize } \alpha= & \sum_{i=1}^{K} \widetilde{C_{i}^{a s f}} x_{i}, \\
= & \min \widetilde{\alpha}=\sum_{i=1}^{5} \widetilde{C_{i}^{a s f}} \circledast x_{j}, \\
= & \left((51.55,56.55,67.55) \circledast x_{1} \oplus(24.5653,29.5653,40.5653) \circledast x_{2},\right. \\
& \oplus(41.24 \\
& \oplus\left((177.892,182.892,193.892) \circledast x_{5}\right.
\end{aligned}
$$


Under the subjected constraint 0.16 , we obtain an infeasible solution after converting into LP objective function by using the weight formula, $\min \tilde{\alpha}^{\vee}=56.25 x_{1}+29.2653 x_{2}+$ $45.94 x_{3}+20.165 x_{4}+182.5921 x_{5}$.

\section{Model-IV(a)}

In this model, we considered cost and demand (right-hand side of constraint equations) a symmetric spherical fuzzy number. The spherical fuzzy energy optimization model for the textile industry according to this model is as follows:

$$
\begin{aligned}
\min \widetilde{\alpha}= & \sum_{j=1}^{m} \widetilde{C_{j}^{s s f}} \circledast x_{j}, \\
= & \left(\widetilde{51.55)^{s s f}} \circledast x_{1},\right. \\
& \oplus\left(2 \widetilde{\sigma_{5653}}\right)^{s s f} \circledast x_{2}, \\
& \oplus\left(\widetilde{4_{1.24}}\right)^{s s f} \circledast x_{3}, \\
& \oplus(\widetilde{15.465})^{s s f} \circledast x_{4}, \\
& \oplus(\widetilde{177.8921})^{s s f} \circledast x_{5},
\end{aligned}
$$

subjected to

$$
\begin{aligned}
x_{1}-x_{2} & \geq(400)^{s s f}, \\
0.03 x_{2}-x_{3}+0.07 x_{4} & =0^{s s f}, \\
0.97 x_{2}-x_{4} & =0^{s s f}, \\
0.93 x_{4}-x_{5} & \geq(600)^{s s f}, \\
0.96 x_{5} & \geq(20000)^{s s f}, \\
0.007 x_{1}-0.007 x_{2}+0.013 x_{4}+0.0062 x_{5} & \leq(720)^{s s f}, \\
x_{i} & \geq 0, \quad i=1,2,3,4,5,
\end{aligned}
$$

converting the above model into LP, we obtain

$\min \widetilde{\alpha^{\gamma}}=55.05 x_{1}+28.0653 x_{2}+44.74 x_{3}+18.965 x_{4}+181.392 x_{5}$,

under the real constraints

$$
\begin{aligned}
x_{1}-x_{2} & \geq 435 \\
0.03 x_{2}-x_{3}+0.07 x_{4} & =0 \\
0.97 x_{2}-x_{4} & =0 \\
0.93 x_{4}-x_{5} & \geq 635 \\
0.96 x_{5} & \geq 20035
\end{aligned}
$$$$
0.007 x_{1}-0.007 x_{2}+0.013 x_{4}+0.0062 x_{5} \leq 755 \text {, }
$$

$$
x_{i} \geq 0, i=1,2,3,4,5 \text {. }
$$

\section{Model-IV(b)}

$$
\begin{aligned}
\min \widetilde{\alpha^{v}}= & \sum_{j=1}^{m} \widetilde{C_{j}^{a s f}} \circledast x_{j}, \\
= & (51.55)^{a s f} \circledast x_{1}, \\
& \oplus(24.5653)^{a s f} \circledast x_{2}, \\
& \oplus(\widetilde{1.24})^{\text {asf }} \circledast x_{3}, \\
& \oplus(15.465)^{a s f} \circledast x_{4}, \\
& \oplus(177.8921)^{a s f} \circledast x_{5},
\end{aligned}
$$

Subjected to

$$
\begin{aligned}
x_{1}-x_{2} & \geq(400)^{a s f}, \\
0.03 x_{2}-x_{3}+0.07 x_{4} & =0^{a s f},
\end{aligned}
$$

subjected to

$$
\begin{aligned}
0.97 x_{2}-x_{4} & =0^{\text {asf }}, \\
0.93 x_{4}-x_{5} & \geq(600)^{\text {asf }}, \\
0.96 x_{5} & \geq(20000)^{a s f}, \\
0.007 x_{1}-0.007 x_{2}+0.013 x_{4}+0.0062 x_{5} & \leq(720)^{a s f}, \\
x_{i} & \geq 0, \quad i=1,2,3,4,5,
\end{aligned}
$$

converting the above model into LP, we obtain

$$
\begin{aligned}
\min \tilde{\alpha^{\gamma}}= & 56.25 x_{1}+29.2653 x_{2}+45.94 x_{3} \\
& +20.165 x_{4}+182.5921 x_{5},
\end{aligned}
$$

under defuzzified constraints

$$
\begin{aligned}
x_{1}-x_{2} & \geq 437.2, \\
0.03 x_{2}-x_{3}+0.07 x_{4} & =0, \\
0.97 x_{2}-x_{4} & =0 \\
0.93 x_{4}-x_{5} & \geq 637.2, \\
0.96 x_{5} & \geq 20037.2, \\
0.007 x_{1}-0.007 x_{2}+0.013 x_{4}+0.0062 x_{5} & \leq 757.2, \\
x_{i} & \geq 0, \quad i=1,2,3,4,5 .
\end{aligned}
$$

Table 4 shows the optimal output we obtained through different models.

\section{Postoptimal Analysis}

In present study eight models with different symmetric and asymmetric spherical fuzzy changes were tried. Out of eight models only five models provided us a feasible solution for spherical fuzzy energy optimization model 0.5 and one of 
TABLE 4: Optimal solutions.

\begin{tabular}{|c|c|c|c|c|c|c|}
\hline Optimization model & Objective value & $x_{1}$ & $x_{2}$ & $x_{3}$ & $x_{4}$ & $x_{5}$ \\
\hline LP & 5987499 & 24195.38 & 23759.38 & 2326.043 & 23046.59 & 20833.33 \\
\hline IFLP & 5927615.1 & 24159.4 & 23759.4 & 874.104 & 23046.6 & 20833.33 \\
\hline Model-I(a) & 5702683 & 21744.58 & 21344.58 & 3639.78 & 21451.3 & 20100.50 \\
\hline Model-I(b) & Infeasible solution & 0 & 0 & 0 & 0 & 0 \\
\hline Model-II(a) & 5717777.8 & 21851.93 & 21416.93 & 3652.12 & 21524.02 & 20135.68 \\
\hline Model-II(b) & Infeasible solution & 0 & 0 & 0 & 0 & 0 \\
\hline Model-III(a) & 6027917.33 & 21851.93 & 21416.93 & 3652.12 & 21524.02 & 20135.68 \\
\hline Model-III(b) & Infeasible solution & 0 & 0 & 0 & 0 & 0 \\
\hline Model-IV(a) & 6333697.02 & 24273.59 & 23838.59 & 2333.8 & 23123.43 & 20869.79 \\
\hline Model-IV(b) & 6448227.44 & 24280.77 & 23843.57 & 2334.28 & 23128.26 & 20872.08 \\
\hline
\end{tabular}

TABLE 5: Model-I(a) sensitivity analysis report.

\begin{tabular}{|c|c|c|c|c|c|}
\hline Variables & Final value & Reduced cost & Objective coefficient & Allowable increase & Allowable decrease \\
\hline$x_{1}$ & 21744.57 & 0 & 51.55 & $1 \mathrm{E}+30$ & 51.55 \\
\hline$x_{2}$ & 21344.57 & 0 & 24.56 & $1 \mathrm{E}+30$ & 98.68 \\
\hline$x_{3}$ & 3639.78 & 0 & 41.24 & $1 \mathrm{E}+30$ & 578.68 \\
\hline$x_{4}$ & 21451.3 & 0 & 15.46 & $1 \mathrm{E}+30$ & 98.19 \\
\hline$x_{5}$ & 20100.50 & 0 & 177.89 & $1 \mathrm{E}+30$ & 279.64 \\
\hline Constraint & Final value & Shadow price & RHS & Allowable increase & Allowable decrease \\
\hline 1 & 400 & 51.55 & 400 & 21923.36 & 21744.57 \\
\hline 2 & $4.55 \mathrm{E}-13$ & -41.24 & 0 & 3639.78 & $1 \mathrm{E}+30$ \\
\hline 3 & 0 & 78.4 & 0 & $1 \mathrm{E}+30$ & 21451.3 \\
\hline 4 & 600 & 101.75 & 600 & 13462.94 & 20700.50 \\
\hline 5 & 20000 & 281.05 & 20000 & 9685.38 & 20000 \\
\hline 6 & 489.80 & 0 & 720 & $1 \mathrm{E}+30$ & 230.19 \\
\hline
\end{tabular}

them provided the best optimal result as compared to all SF models and LP as shown in Table 4 while all the other spherical fuzzy models resulted those objective values, which are much higher than real LP. Overall, if we combine the objective value in the form of inequality keeping LP greatest we get:

$$
\text { SSFModel }-I(a)<\text { SSFModel }-I I(a)<\text { IFLP }<\text { LP, }
$$

the objective values obtained from these methods also satisfy the relation as

$$
5702683<5717777.8<5927615.1<5933824<5987499 \text {. }
$$

Except one ASF model, all the other ASF (models-I, II, III) energy optimization models gave "Infeasible solution" in 0.5 and one ASF model-IV, which provided a solution that have the most highest optimal value (greater than LP) where objective function was needed to minimize, whereas in 0.3 where the objective function was needed to maximize the similar models, ASF (models-I, II, III) provided feasible solution, but other than SSF and AS (models- I, II), all the other remaining models provided outputs less than LP; that is, the outputs of these models are much lesser than LP's outputs. Here, in the following tables, the sensitivity report and limits of all those SF models in 0.5 are discussed whose optimal solutions were not greater than the LP textile energy model.
In Table 5 the flexibility regarding allowable change in optimal and feasible conditions is mentioned. Since solution is effected by both and impacts the efficiency of model, how much cost and demand fluctuation can be handled by these models in 0.5 is discussed, whereas in Tables 6, 7 and 8 the feasibility range of the decision variable is discussed along with the optimal solution between that range. In Table 9 the validity range of all the parameters and decision variables of the best optimal model for 0.5 is mentioned. According to the above postoptimal analysis, we obtain the following information about the range of each factor of the energy optimization model in a spherical environment. SSF model-I(a) is providing a minimal optimal value of the objective function for a longer range of coefficients that insure the feasibility for a huge change in 0.5 . In both situations, where objective function is needed to maximize 0.3 or to minimize 0.5 , SSF model-I(a) provided the best results.

From Figure 2 it is clearly seen that all the decision variables in SSF model-I for 0.5 is having least value as compared to other models except $x_{3}$. This $x_{3}$ is providing its minimal value in the intuitionistic fuzzy environment.

In Figure 3 an objective value of 0.5 in different fuzzy and LP environments is graphically presented. Three bars in the right-hand side denote ASF models and are clearly higher than even LP's bar. Therefore, they are not considered the best models for 0.5 . SSF model-I is providing the best results as compared to others. 
Table 6: Model-I(a) limits report.

\begin{tabular}{lccccc}
\hline Variable & Values & Lower limit & Objective result & Upper limit & Objective result \\
\hline$x_{1}$ & 21744.57 & 21744.57 & 5702683.001 & 43667.94 & 6832832.271 \\
$x_{2}$ & 21344.57 & 21344.57 & 5702683.001 & 21344.57 & 5702683.001 \\
$x_{3}$ & 3639.78 & 3639.78 & 5702683.001 & 3639.78 & 5702683.001 \\
$x_{4}$ & 21451.3 & 21451.3 & 5702683.001 & 21451.30 & 5702683.001 \\
$x_{5}$ & 20100.50 & 20100.50 & 5702683.001 & 20100.50 & 5702683.001 \\
\hline
\end{tabular}

TABle 7: Model-II(a) sensitivity analysis report.

\begin{tabular}{lccccc}
\hline Variables & Final value & Reduced cost & Objective coefficient & Allowable increase & Allowable decrease \\
\hline$x_{1}$ & 21851.93 & 0 & 51.55 & $1 \mathrm{E}+30$ & 51.55 \\
$x_{2}$ & 21416.93 & 0 & 24.56 & $1 \mathrm{E}+30$ & $1 \mathrm{E}+30$ \\
$x_{3}$ & 3652.12 & 0 & 41.24 & $1 \mathrm{E}+30$ & 58.68 \\
$x_{4}$ & 21524.02 & 0 & 15.46 & $1 \mathrm{E}+30$ & 98.68 \\
$x_{5}$ & 20135.68 & 0 & 177.89 & Allowable increase & Allowable decrease \\
\hline Constraint & Final value & Shadow price & RHS & 25085.47 & 21851.9 \\
\hline 1 & 435 & 51.55 & 435 & 3652.12 & $1 \mathrm{E}+30$ \\
2 & $4.55 \mathrm{E}-13$ & -41.24 & 0 & $1 \mathrm{E}+30$ & 21524.02 \\
3 & 0 & 78.4 & 635 & 15404.76 & 20770.68 \\
4 & 635 & 101.75 & 20035 & 11082.36 & 20035 \\
5 & 20035 & 281.045 & 755 & $1 \mathrm{E}+30$ & 263.4 \\
\hline
\end{tabular}

TABle 8: Model-II(a) limits report.

\begin{tabular}{lccccc}
\hline Variable & Values & Lower limit & Objective result & Upper limit & Objective result \\
\hline$x_{1}$ & 21851.93 & 21851.93 & 5717777.832 & 46937.41 & 7010934.09 \\
$x_{2}$ & 21416.93 & 21416.93 & 5717777.832 & 21416.93 & 5717777.832 \\
$x_{3}$ & 3652.12 & 3652.12 & 5717777.832 & 3652.12 & 5717777.832 \\
$x_{4}$ & 21524.02 & 21524.02 & 5717777.832 & 21524.02 & 5717777.832 \\
$x_{5}$ & 20135.68 & 20135.68 & 5717777.832 & 20135.69 & 5717777.832 \\
\hline
\end{tabular}

TABLE 9: Validity range of SSF-I(a).

\begin{tabular}{lcc}
\hline Factors & SSF model-I(a) & Range \\
\hline$x_{1}$ & 21744.56 units & 21923.38 unit \\
$x_{2}$ & 21344.56 units & 0 \\
$x_{3}$ & 3539.73 units & 0 \\
$x_{4}$ & 21451.3 units & 0 unit \\
$x_{5}$ & 20100 units & 0 unit \\
$c_{1}$ & $51.55 \mathrm{Rs} . /$ unit & $51.55 \mathrm{Rs} / \mathrm{unit}$ \\
$c_{2}$ & $24.56 \mathrm{Rs} / \mathrm{units}$ & $98.68 \mathrm{Rs} / \mathrm{unit}$ \\
$c_{3}$ & $41.24 \mathrm{Rs} / \mathrm{units}$ & $578.71 \mathrm{Rs} / \mathrm{unit}$ \\
$c_{4}$ & $15.46 \mathrm{Rs} . / \mathrm{units}$ & $98.2 \mathrm{Rs} / \mathrm{unit}$ \\
$c_{5}$ & $177.89 \mathrm{Rs} . / \mathrm{units}$ & $279.64 \mathrm{Rs} / \mathrm{unit}$ \\
$d_{1}$ & $400 \mathrm{~kg} / \mathrm{month}$ & $43667 \mathrm{~kg} / \mathrm{month}$ \\
$d_{2}$ & 0 & $3639.78 \mathrm{~kg} / \mathrm{month}$ \\
$d_{3}$ & 0 & $21451.3 \mathrm{~kg} / \mathrm{mon}$ th \\
$d_{4}$ & $20000 \mathrm{~kg} / \mathrm{month}$ & $29685.39 \mathrm{~kg} / \mathrm{month}$ \\
$d_{5}$ & $600 \mathrm{~kg} / \mathrm{month}$ & $43163.44 \mathrm{~kg} / \mathrm{month}$ \\
$d_{6}$ & 720 working hours $/ \mathrm{month}$ & $489.81 \mathrm{hours} / \mathrm{month}$ \\
\hline
\end{tabular}


COMPARISON OF DECISION VARIABLS IN DIFFERENT OPTIMIZATION

ENVIRONMENT

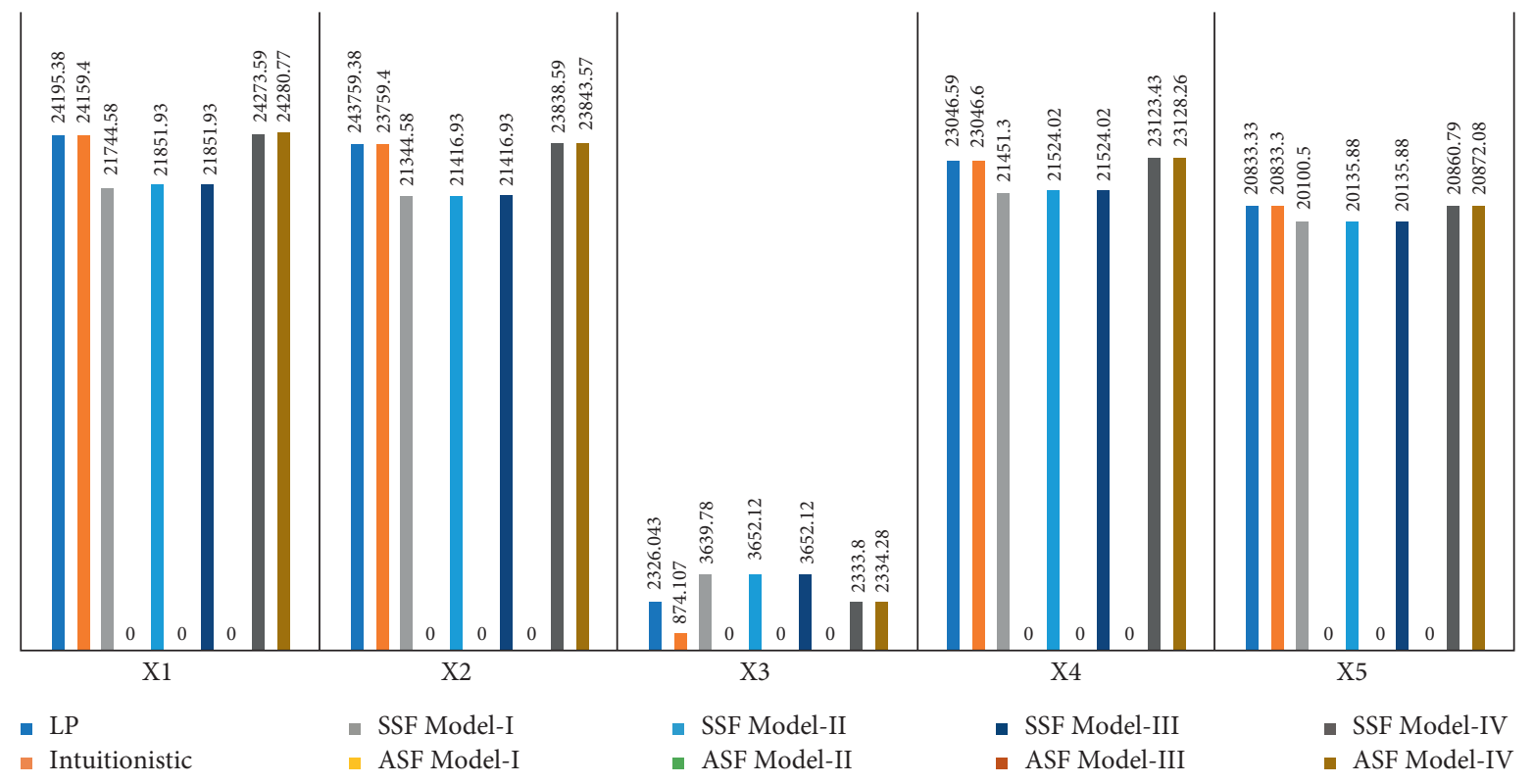

Figure 2: Comparison of decision variables.

COMPARISON OF OPTIMAL SOLUTION

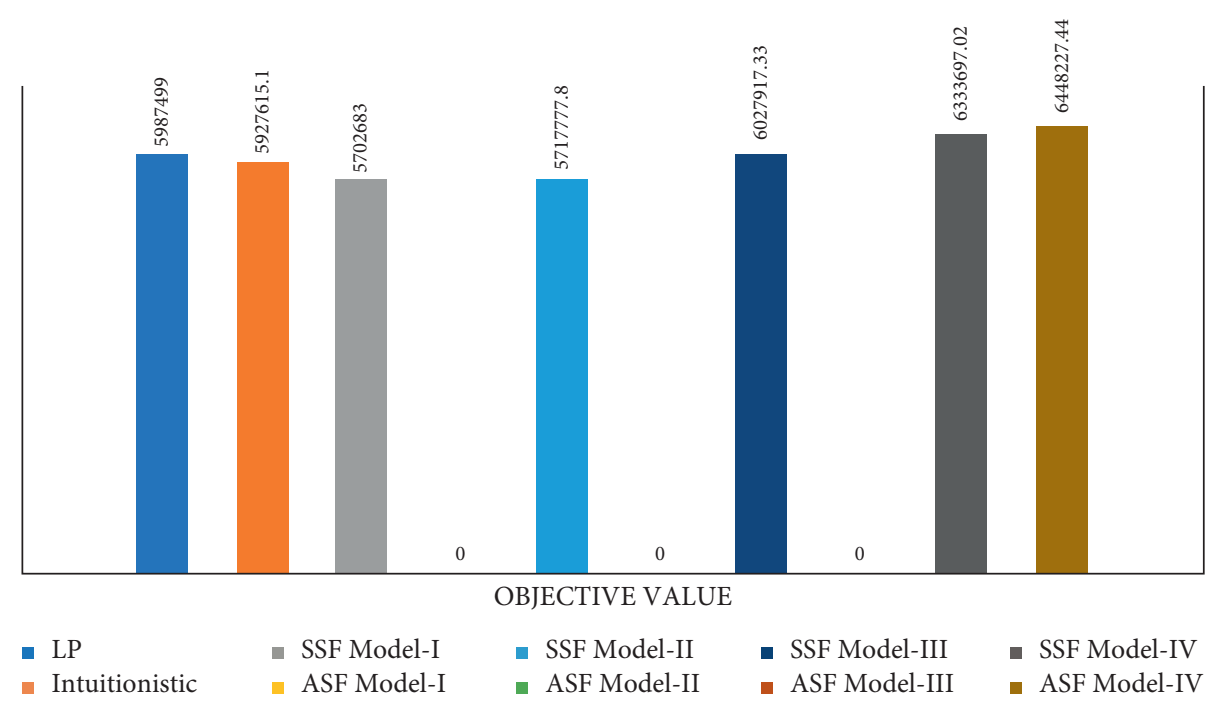

Figure 3: Comparison of objective function values. 

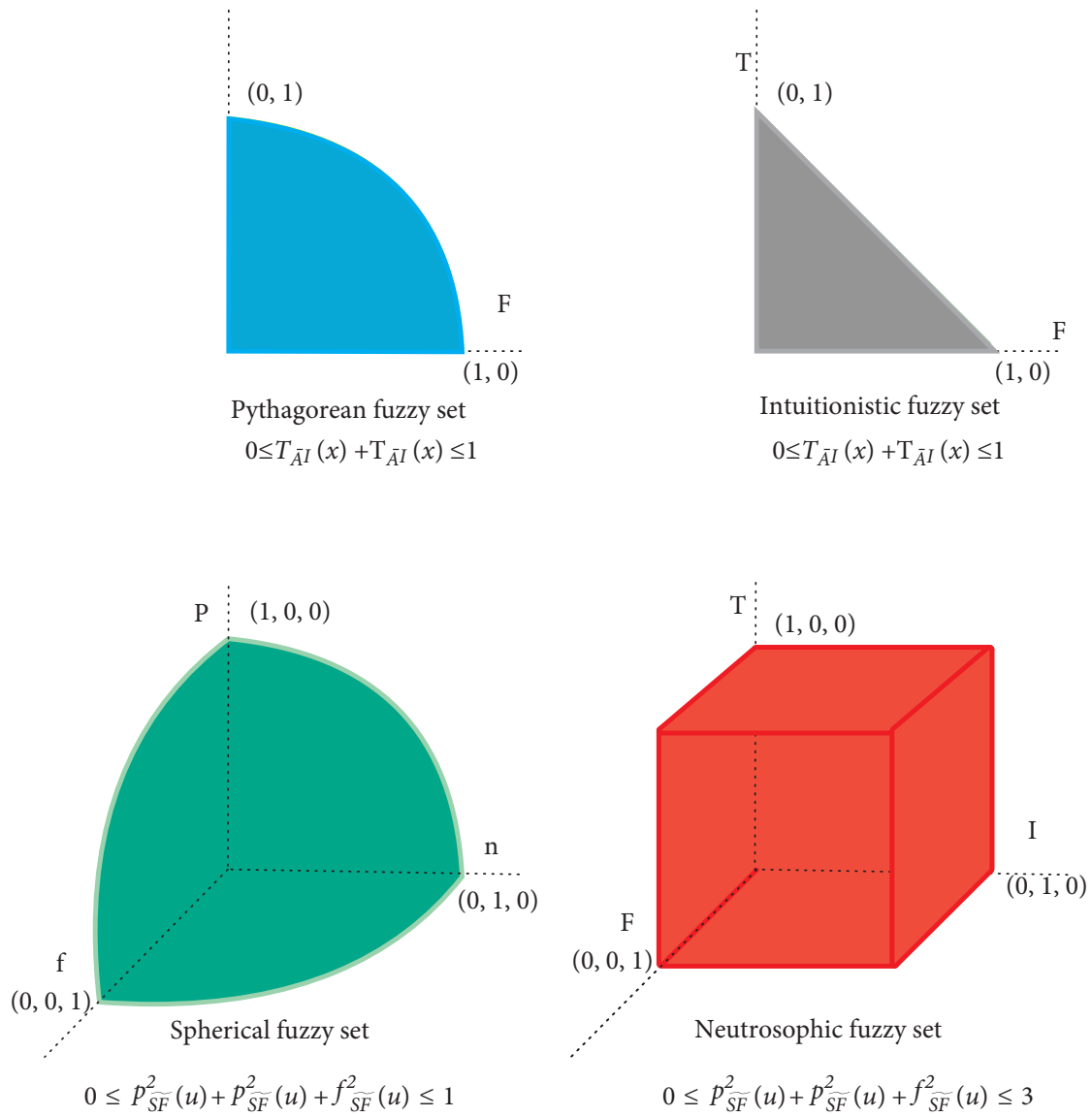

Figure 4: Comparison of SF with other fuzzy sets.

\section{Conclusion}

Spherical fuzzy set (SFS) is a well-known generalization of widely studied fuzzy sets, and significant research has been carried out to investigate SFS set theocratic properties and applications in other fields. Due to the involvement of positive, neutral, and negative grades, SFS can handle uncertainties better than fuzzy sets. In this article, linear optimization is carried out by utilizing spherical fuzzy numbers. The method is superior to previously defined techniques as shown in.

(1) Due to the spherical region, parameters are highly flexible and can provide optimal solution between a long range

(2) Spherical fuzzy set is a super set of the intuitionistic and Pythagorean fuzzy set, so it will cover more area graphically (see Figure 4) and can easily target those points for solution that are far away to those points, which are obtained through LP or IF technique

(3) No need to construct or change a model for a huge change due to long-range flexibility of parameters

The method can be used in any decision-making problem simply by identifying the objectives, parameters, and the constraints imposed to maximize or minimize the objective. In recent years, the fuzzy set and its generalizations are used widely in decision-making related to real- life problems $[22,23]$. Any advancement in basic fuzzy set theocratic concepts will ultimately improve the accuracy of its implementation by incorporating the imprecision and vagueness in the data. Spherical fuzzy optimization techniques can be used to find the suitable weights for the best criteria over others in the decision-making process. [24-26].

\section{Data Availability}

No data were used to support this study.

\section{Conflicts of Interest}

The authors declare that there are no conflicts of interest regarding the publication of this article.

\section{Authors' Contributions}

All authors contributed equally to the preparation of this manuscript.

\section{Acknowledgments}

The fifth author (YUG) would like to acknowledge that this publication was made possible by a grant from the Carnegie Corporation of New York. 


\section{References}

[1] R. Q. Wang, L. Jiang, Y. D. Wang, and A. P. Roskilly, "Energy saving technologies and mass-thermal network optimization for decarbonized iron and steel industry: a review," Journal of Cleaner Production, vol. 274, Article ID 122997, 2020.

[2] I. Ullah, Z. Khitab, M. Khan, and S. Hussain, "An efficient energy management in office using bio-inspired energy optimization algorithms," Processes, vol. 7, no. 3, Article ID 142, 2019.

[3] E. Ozturk, N. C. Cinperi, and M. Kitis, "Improving energy efficiency using the most appropriate techniques in an integrated woolen textile facility," Journal of Cleaner Production, vol. 254, Article ID 120145, 2020.

[4] I. Kimutai, P. Maina, and A. Makokha, "Energy optimization model using linear programming for process industry: a case study of textile manufacturing plant in Kenya," International Journal of Energy Engineering, vol. 9, no. 2, pp. 45-52, 2019.

[5] https://invest.gov.pk/textilepakistan, 2021.

[6] K. Mustafa, "Despite go-ahead by PM twice: textile Policy 2020-25 in the doldrums," Available at: https://Thenews.com. pk, Jan 242021.

[7] L. V. Kantorovich, "Mathematical methods of organizing and planning production," Management Science, vol. 6, no. 4, pp. 366-422, 1960.

[8] L. A. Zadeh, "Fuzzy sets," Information and Control, vol. 8, no. 3, pp. 338-353, 1965.

[9] H.-J. Zimmermann, "Fuzzy programming and linear programming with several objective functions," Fuzzy Sets and Systems, vol. 1, no. 1, pp. 45-55, 1978.

[10] K. T. Atanassov, "Intuitionistic fuzzy sets," Fuzzy Sets and Systems, vol. 20, no. 1, pp. 87-96, 1986.

[11] P. P. Angelov, "Optimization in an intuitionistic fuzzy environment," Fuzzy Sets and Systems, vol. 86, no. 3, pp. 299-306, 1997.

[12] R. R. Yager, "Pythagorean fuzzy subsets," in 2013 joint IFSA world congress and NAFIPS annual meeting (IFSA/NAFIPS), pp. 57-61, Edmonton, AB, Canada, 24-28 June 2013.

[13] F. Smarandche, A Unifying Field in Logics: Neutrosophic Logic, American Press, Reston, Virginia, Philosophy, 1999.

[14] F. Ahmad, A. Y. Adhami, and F. Smarandache, "Neutrosophic optimization model and computational algorithm for optimal shale gas water management under uncertainty," Symmetry, vol. 11, no. 4, p. 544, 2019.

[15] F. Ahmad, A. Y. Adhami, and F. Smarandache, "Modified neutrosophic fuzzy optimization model for ooptimal closedloop supply chain management under uncertainity," Optimization Theory Based on Neutrosophic and Plithogenic Sets, vol. 11, no. 4, pp. 343-403, 2020.

[16] G. F. Kutlu and C. Kahraman, "Spherical fuzzy sets and spherical fuzzy TOPSIS method," Journal of Intelligent and Fuzzy Systems, vol. 36, no. 4, pp. 337-352, 2019.

[17] F. Ahmad and A. Y. Adhami, "Spherical fuzzy linear programming problem," Decision Making with Spherical Fuzzy Sets, vol. 392, pp. 455-472, 2021.

[18] A. Rafiq, S. Ashraf, S. Abdullah, T. Mahmood, and S. Muhammad, "The cosine similarity measure of spherical fuzzy sets and their applications in decision making," Journal of Intelligent and Fuzzy Systems, vol. 36, no. 8, pp. 1-15, 2019.

[19] F. Ahmad and A. Y. Adhami, "Neutrosophic programming approach to multiobjective nonlinear transportation problem with fuzzy parameters," International Journal of Management Science and Engineering Management, vol. 14, no. 3, pp. 218-229, 2019.
[20] https://www.nepra.org, 2021.

[21] http://www.ogra.org, 2021.

[22] M. A. Alsalem, R. Mohammed, O. S. Albahri et al., "Rise of multiattribute decision making in combating COVID-19: a systematic review of the state of the art literature," International Journal of Intelligent Systems, 2021.

[23] A. S. Albahri, A. A. Zaidan, O. S. Albahri et al., "Development of IoT-based mhealth framework for various cases of heart disease patients," Health Technology, vol. 11, no. 5, pp. 1013-1033, 2021.

[24] A. S. Albahri, O. S. Albahri, A. A. Zaidan et al., "Integration of fuzzy-weighted zero-inconsistency and fuzzy decision by opinion score methods under a q-rung orthopair environment: a distribution case study of COVID-19 vaccine doses," Computer Standards \& Interfaces, vol. 80, Article ID 103572, 2022.

[25] O. S. Albahri, A. A. Zaidan, M. M. Salih et al., "Multidimensional benchmarking of the active queue management methods of network congestion control based on extension of fuzzy decision by opinion score method," International Journal of Intelligent Systems, vol. 36, no. 2, pp. 796-831, 2021.

[26] M. A. Alsalem, H. A. Alsattar, A. S. Albahri et al., "Based on T-spherical fuzzy environment: a combination of FWZIC and FDOSM for prioritising COVID-19 vaccine dose recipients," Journal of Infection and Public Health, vol. 14, no. 10, pp. 1513-1559, 2021. 